\title{
Technical Challenges and Scientific Payoffs of Muon Beam Accelerators for Particle Physics
}

\author{
Michael S. Zisman
}

\begin{abstract}
Progress in particle physics has largely been determined by development of more capable particle accelerators. This trend continues today with the recent advent of high-luminosity electron-positron colliders at KEK and SLAC operating as "B factories," the imminent commissioning of the Large Hadron Collider at CERN, and the worldwide development effort toward the International Linear Collider. Looking to the future, one of the most promising approaches is the development of muon-beam accelerators. Such machines have very high scientific potential, and would substantially advance the state-of-the-art in accelerator design. A $20-50 \mathrm{GeV}$ muon storage ring could serve as a copious source of well-characterized electron neutrinos or antineutrinos (a Neutrino Factory), providing beams aimed at detectors located $3000-7500 \mathrm{~km}$ from the ring. Such long baseline experiments are expected to be able to observe and characterize the phenomenon of chargeconjugation-parity (CP) violation in the lepton sector, and thus provide an answer to one of the most fundamental questions in science, namely, why the matter-dominated universe in which we reside exists at all. By accelerating muons to even higher energies of several TeV, we can envision a Muon Collider. In contrast to composite particles like protons, muons are point particles. This means that the full collision energy is available to create new particles. A Muon Collider has roughly ten times the energy reach of a proton collider at the same collision energy, and has a much smaller footprint. Indeed, an energy frontier Muon Collider could fit on the site of an existing laboratory, such as Fermilab or BNL. The challenges of muon-beam accelerators are related to the facts that $i$ ) muons are produced as a tertiary beam, with very large 6D phase space, and ii) muons are unstable, with a lifetime at rest of only $2 \mu \mathrm{s}$. How these challenges are accommodated in the accelerator design will be described. Both a Neutrino Factory and a Muon Collider require large numbers of challenging superconducting magnets, including large aperture solenoids, closely spaced solenoids with opposing fields, shielded solenoids, very high field $(\sim 40-50 \mathrm{~T})$ solenoids, and storage ring magnets with a room-temperature midplane section. Uses for the various magnets will be outlined, along with R\&D plans to develop these and other required components of such machines.
\end{abstract}

Index Terms-Accelerator cavities, Accelerator magnets, Particle beams, Solenoids.

\section{INTRODUCTION}

$\mathrm{H}$ ISTORICALLY, particle physics has depended on advances in accelerator design to make scientific progress. This

Manuscript received September 25, 2007. This work was supported by the U.S. Department of Energy, Office of High Energy Physics, under contract number DE-AC02-05CH11231.

Michael S. Zisman is with the Accelerator \& Fusion Research Division, Lawrence Berkeley National Laboratory, Berkeley, CA 94720 USA (phone: 510-486-5765; fax: 510-486-5788; e-mail: mszisman@lbl.gov). trend started with electrostatic accelerators (Cockcroft-Walton and van de Graaff devices) and continued with cyclotrons, synchrocyclotrons, synchrotrons, and most recently colliders (both circular and linear). Advances in accelerator performance come only with corresponding advances in accelerator technology, including magnets, vacuum systems, RF systems, feedback systems, diagnostic systems, etc.

In the early days of accelerators, building the accelerator and building the detectors were not considered separate tasks, that is, such activities were simply part of preparing the experiment. Modern accelerators - and their technical subsystems - require a high degree of specialization, and experts in all aspects are needed.

\section{PARTICle Physics Questions}

Particle physics is a broad subject, and it is neither practical nor desirable to try to do it justice here. From the perspective of accelerator-related efforts, there are two main thrusts. First, we desire to gain an understanding of the origins of the masses of the fundamental particles. These cover a huge range. The recently observed top quark has a mass of about 171,000 $\mathrm{MeV} / \mathrm{c}^{2}$, nearly the mass of a gold nucleus, and the $\mathrm{Z}$ and $\mathrm{W}$ bosons, carriers of the weak force, have masses of 91,000 and $80,000 \mathrm{MeV} / \mathrm{c}^{2}$ (comparable to a mid-range nucleus). At the other end of the spectrum, neutrinos are nearly massless (and indeed were thought until recently to be exactly massless) with a mass in the neighborhood of $0.000001 \mathrm{MeV} / \mathrm{c}^{2}$.

The second main topic is to understand why we live in a matter-dominated universe. In essence, this question asks why we are all here, so it is pretty fundamental from a provincial viewpoint. At the time of the Big Bang, equal amounts of matter and anti-matter were created. In a symmetric universe, all of the matter and anti-matter would have annihilated. The reason it did not is believed to be due to a slight asymmetry in reaction rates between matter and anti-matter, referred to as "charge-conjugation-parity" $(\mathrm{CP})$ violation.

$\mathrm{CP}$ violation is a well-known phenomenon in the quark sector, having been observed in the $\mathrm{K}$ meson system more than 40 years ago [1]. In the past 10 years, the study of $\mathrm{CP}$ violation has centered on the heavier $\mathrm{B}$ meson system. Two high luminosity "B factories" have been built, PEP-II [2] at SLAC and KEKB [3] at KEK. Both of these machines have reached a luminosity in excess of $1 \times 10^{34} \mathrm{~cm}^{-2} \mathrm{~s}^{-1}$. Results to date have shown that the observed $\mathrm{CP}$ violation is consistent with Standard Model predictions, and thus is insufficient to explain the observed baryon asymmetry in the universe. The prevalent view is that the required additional $\mathrm{CP}$ violation 
must have been due to the lepton sector. $\mathrm{CP}$ violation in the lepton sector has never been observed, but neutrinos are considered a good candidate to exhibit the effect.

\section{ACCELERATOR DELIVERABLES}

Particle accelerators are designed to deliver two parameters to the user-energy and luminosity. Of these, energy is by far the easier parameter to deliver. In practice, the two deliverables are often, but not always, interchangeable. At the LHC [4], where beam commissioning will begin in mid-2008, the discovery reach can be enhanced either by increasing the beam energy (an upgrade referred to as "DLHC") or by increasing the luminosity (an upgrade referred to as "SLHC"). In general, an energy increase is easier for the experimenters to accommodate. Substantially higher luminosity invariably requires significant upgrades to the detector capabilities, which are themselves expensive and sometimes risky. A substantial energy increase requires replacing or upgrading all of the ring dipoles, and perhaps other magnets as well. A luminosity upgrade typically requires fewer new magnets, but they are the most challenging magnets to design and build.

Luminosity, $L$, is a measure of the collision rate per unit area. If the probability for a particular event type (quantified by its "cross section" in units of $\mathrm{cm}^{2}$ ) is denoted $\sigma$, then the event rate, $R$, for that event type is given by

$$
R=L \sigma
$$

where $L$ is in units of $\mathrm{cm}^{-2} \mathrm{~s}^{-1}$. In practice, it is the integrated luminosity, $\int L d t$, that is the figure-of-merit for looking at rare events. Integrated luminosity is often quoted in units of inverse barns, where $1 \mathrm{~b}^{-1}=10^{24} \mathrm{~cm}^{-2}$. For today's colliders, integrated luminosity might be quoted in units of $\mathrm{pb}^{-1}, \mathrm{fb}^{-1}$, and, before much longer, the first data set representing $1 \mathrm{ab}^{-1}$ will be available. For a peak luminosity of $1 \times 10^{34} \mathrm{~cm}^{-2} \mathrm{~s}^{-1}$ and a "year" of $10^{7} \mathrm{~s}$, the integrated luminosity would be 100 $\mathrm{fb}^{-1}$.

For a collider with equal beam sizes at the interaction point (IP), the luminosity is given by

$$
L=\frac{N_{1} N_{2} f_{c}}{4 \pi \sigma_{x}^{*} \sigma_{y}^{*}}
$$

where $N_{1}$ and $N_{2}$ are the numbers of particles in the two colliding beams, $f_{\mathrm{c}}$ is the collision frequency, and $\sigma_{x}{ }^{*}$ and $\sigma_{y}{ }^{*}$ are the horizontal and vertical beam sizes, respectively, at the IP. To increase the luminosity of a collider, we must design for more intense beams and smaller beam sizes at the IP, both of which are challenging.

\section{TODAY'S MACHINES}

High energy physics experiments typically make use of circular colliders, which deliver counter-rotating beams that collide at one or more IPs. Colliders are separated into two broad categories, hadron colliders (protons, antiprotons, heavy ions) and lepton colliders (electrons and positrons). Until recently, most circular colliders were single-ring devices, that is, both beams circulated in a common magnetic field and a common vacuum chamber. In this configuration, the two beams had to be of opposite charge and beams of particles and their corresponding anti-particles were utilized, e.g., electrons and positrons or protons and antiprotons.

To get higher luminosity, modern colliders [2]-[5] use two separate rings, and thus no longer require the beams to have opposite sign. Use of a two-ring collider decouples the beams completely ${ }^{1}$, and permits beams of different magnetic rigidity to be used, though there are typically rather complicated constraints on the design of the common IP optics.

\section{A. Machine Limitations of Hadron Colliders}

The most serious limitation of hadron colliders is that protons are composite particles, comprising quarks and gluons. Thus, only about $10 \%$ of the beam energy is available for the "hard" collisions that can lead to production of new particles. In effect, a $10 \mathrm{TeV}$ hadron collider is required to probe the $1 \mathrm{TeV}$ mass scale.

Another limitation stems from the difficulty in producing antiprotons. The available antiproton intensity tends to limit the peak luminosity, and the slow production rate means that it takes many hours to replace them if the beam is lost, thereby reducing the integrated luminosity.

The use of a proton-proton collider bypasses the second limitation, but not the first one.

\section{B. Machine Limitations of Lepton Colliders}

The main challenge of present lepton colliders is dealing with the emitted power from synchrotron radiation. The power in $\mathrm{kW}$ emitted by electrons or positrons in a circular collider is given by:

$P_{S R}=\frac{88.5 E^{4} I}{\rho}$

where $E$ is the beam energy in $\mathrm{GeV}, I$ is the beam current in $\mathrm{A}$, and $\rho$ is the bending radius in the ring dipoles, in $\mathrm{m}$. For a 1 $\mathrm{TeV}$ center-of-mass (c.m.) collider in the LHC tunnel $(C=27$ $\mathrm{km}$ ), a $1 \mathrm{~mA}$ beam would produce roughly $2 \mathrm{GW}$ of radiated power. Operating such an accelerator would require replenishing the lost beam power with an RF system and also removing this much power from the vacuum chamber walls. Both of these requirements are impractical to meet.

For this reason, the favored approach for a high-energy lepton collider is a linear collider, such as the International Linear Collider (ILC) [6]. Although a linear collider avoids the synchrotron radiation limitation, it nonetheless has some drawbacks. Single-pass acceleration is expensive because there is no reuse of the RF hardware, and the facility size is too large (31 km in length for the ILC) to fit on an existing laboratory site.

\section{MuON BEAM ACCELERATORS}

\section{A. Advantages}

Muon beam accelerators have the desirable feature that they can address both of the outstanding accelerator-related questions in particle physics. For the neutrino sector, we create

\footnotetext{
${ }^{1}$ One hybrid collider, HERA [5], has collided electrons with protons, demonstrating the great flexibility of the two-ring collider approach.
} 
beams of neutrinos via the decays:

$$
\mu^{+} \rightarrow e^{+} v_{e} \bar{v}_{\mu}
$$

and

$$
\mu^{-} \rightarrow e^{-} \bar{v}_{e} v_{\mu}
$$

whose kinematical properties are well known and which have minimal hadronic uncertainties in the spectrum and flux. In both cases, the oscillation of an electron neutrino to a muon neutrino gives rise to easily detected "wrong-sign" muons in the detector. (Here, "wrong-sign" means that one observes $\mu^{-}$ from a stored $\mu^{+}$beam, and vice versa.)

Because muons are point particles, all of the muon beam energy is available for producing new particles. Moreover, synchrotron radiation is suppressed compared with the electron case by a factor of the fourth power of the mass ratio, making its effects negligible.

\section{B. Neutrino Factory Capabilities}

Comparative studies to date [7] indicate substantial advantages for a Neutrino Factory over other technical approaches. There are two main unanswered questions in the neutrino sector: $i$ ) is the mass hierarchy "normal," (i.e., the small solar mass splitting is between the two low mass neutrinos) or "inverted;" (i.e., the solar mass splitting is between the two high mass neutrinos); and ii) is there evidence for CP violation. As discussed in [7], especially for the case where the mixing angle $\sin ^{2} \theta_{13}$ is small, a Neutrino Factory is estimated to be the preferred instrument to investigate both questions.

\section{Muon Collider Capabilities}

As noted already in Section V-A, a Muon Collider could easily fit on an existing laboratory site. This is illustrated in Fig. 1, which shows a possible Muon Collider layout on the Fermilab site. The suppression of synchrotron radiation in a muon beam facility leads to a relatively small energy spread, which is of great benefit for making precision studies at the energy frontier. In other words, a Muon Collider can serve simultaneously as a discovery machine and a precision machine at the energy frontier.

\section{Muon Beam Challenges}

Although muons offer many potential physics benefits, their use brings substantial complications as well. Indeed, if intense muon beams were easy to produce, they would already be available.

Firstly, muons are created as a tertiary beam. The proposed production scheme uses a proton beam to bombard a high- $Z$ target. This produces pions, which are captured in a solenoidal decay channel, where they decay to muons. To produce an acceptably large sample of muons, a multi-MW proton beam is required; a typical Neutrino Factory specification is for a 4MW proton driver. A target system capable of tolerating such an intense beam is a substantial challenge.

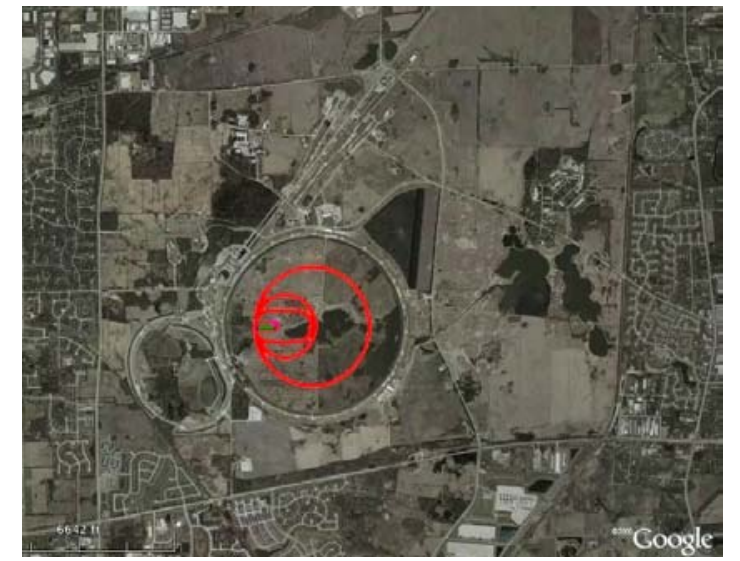

Fig. 1. Aerial view of Fermilab site indicating possible footprint for a Muon Collider facility.

The capture and decay process just described gives rise to a muon beam having a large energy spread and a large transverse phase space. The large transverse phase space has several implications:

1. it favors the use of solenoidal focusing in the lowenergy portions of the facility, as opposed to the more conventional quadrupole focusing. (A solenoid focuses in both planes simultaneously, avoiding the excessively large beam size in one plane when using an alternating polarity quadrupole channel.);

2. it requires a rapid mechanism for reducing the emittance to more tractable values;

3. it requires a high-acceptance acceleration system and decay ring.

The second major challenge of muon beams is due to the short lifetime of the muon, only $2.2 \mu$ s at rest. Clearly, the short lifetime puts a premium on very rapid beam manipulations. A fast emittance cooling technique, "ionization cooling," is needed to reduce the transverse emittance of the muon beam, along with a very rapid acceleration system. As will be discussed in Section VI, the presently untested ionization cooling technique requires high-gradient normal conducting RF cavities due to the need to immerse the cavities in a strong solenoidal magnetic field.

Finally, the decays of the muons lead to potentially severe backgrounds in the detector of a Muon Collider.

There are also a number of challenges related to the magnet requirements:

- In the target area, the initial capture magnet is a 20 T hybrid design.

- In the cooling channel, large aperture magnets, up to $1.5-\mathrm{m}$ diameter, are utilized.

- In the acceleration system, solenoids with very low fringe fields are needed to permit operation of nearby superconducting RF cavities.

- In the acceleration system and decay ring, special split-midplane or shielded dipoles are needed to accommodate the high heat load from muon decay electrons. 
- In the final cooling stages of a Muon Collider, very high strength solenoids, up to $\approx 50 \mathrm{~T}$, are required.

\section{IONIZATION COOLING}

\section{A. 4D Cooling}

Ionization cooling is functionally similar to the more familiar synchrotron radiation damping process. Each process comprises an energy loss mechanism and an energy gain mechanism. The energy loss mechanism in the synchrotron radiation case is just the emission of synchrotron radiation photons, whereas for ionization cooling the loss mechanism is ionization energy loss $(d E / d x)$ in an absorber. In either case, the loss mechanism decreases the particle momentum in all three planes $\left(p_{x}, p_{y}, p_{z}\right)$. For both the synchrotron radiation and ionization cooling cases, the longitudinal momentum, $p_{z}$, is restored with RF cavities. Repeating this process many times, as illustrated schematically in Fig. 2, reduces $p_{x}, p_{y} / p_{z}$, and hence provides $4 \mathrm{D}$ cooling (in $x, p_{x}, y, p_{y}$ phase space).

There is also a heating term in each process - quantum excitation in the synchrotron radiation case and multiple scattering in the case of ionization cooling. As a result, for a given ionization cooling channel design, there is an equilibrium emittance value. To achieve a low equilibrium emittance, the channel should be strongly focusing at the absorber, and the absorber material should have a large radiation length and large value for $d E / d x$. Based on the latter two criteria, hydrogen is by far the best absorber material.

Although the principle of ionization cooling is quite straightforward, the implementation is not. In particular, the need to operate liquid hydrogen absorbers in close proximity with RF cavities is an engineering challenge. As will be seen below, this challenge has been successfully dealt with in the Muon Ionization Cooling Experiment (MICE).

\section{B. $6 D$ Cooling}

To accomplish 6D cooling, it is necessary to add emittance exchange to the mix. The concept, illustrated schematically in Fig. 3, is again straightforward. A system being proposed to accomplish this [8] makes use of solenoids whose centers are displaced to follow the helical central trajectory of the muon beam. The resultant field has both solenoidal and helical dipole components. Parameters are summarized in Table I. An experiment, MANX [9], is being planned at Fermilab to examine the behavior of a helical cooling channel.

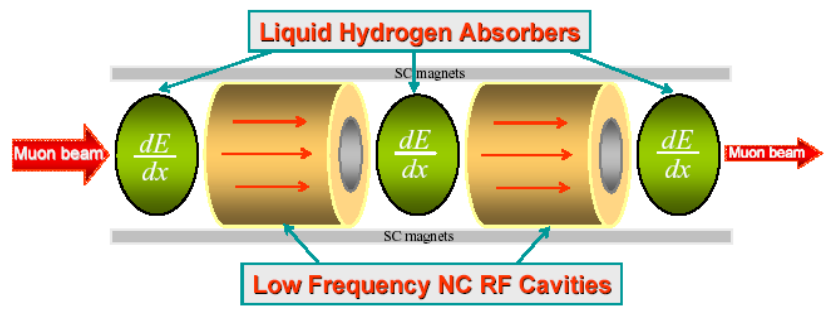

Fig. 2. Schematic of a muon ionization cooling system comprising $\mathrm{LH}_{2}$ absorbers interspersed with $201 \mathrm{MHz}$ RF cavities. The channel is immersed in a strong solenoidal field to contain the beam within the cooling channel.

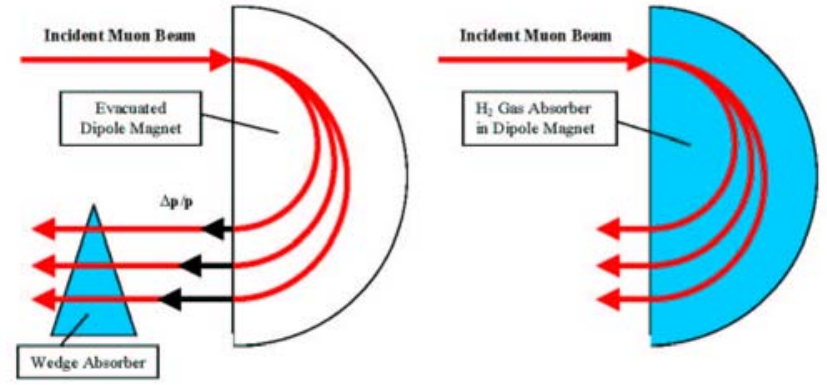

Fig. 3. Schematic illustration of emittance exchange. (Left) a dipole is used to create a dispersive region, i.e., a region where the position of a particle depends on its momentum. A discrete wedge-shaped absorber is placed such that lower energy particles lose less energy and higher energy particles lose more energy, thus reducing the energy spread in the beam. (Right) A gasfilled distributed absorber system to accomplish emittance exchange. High energy particles have a longer path length in the gas and thus lose more energy than do low energy particles, which have a shorter path length.

TABLE I. HeLICAL COOLING ChanNel MaIn PARAmeTERS

\begin{tabular}{lc}
\multicolumn{1}{c}{ Parameter } & Value \\
\hline \hline Inner bore diameter $(\mathrm{m})$ & 0.5 \\
Helical solenoid length (m) & 3.2 \\
Helix twist pitch (m) & 1.6 \\
Beam reference orbit radius (m) & 0.255 \\
Initial dipole flux density $(\mathrm{T})$ & 1.25 \\
Dipole field gradient, $\partial B / \partial z(\mathrm{~T} / \mathrm{m})$ & -0.17 \\
Initial quadrupole flux density, $\partial B / \partial r(\mathrm{~T} / \mathrm{m})$ & -0.88 \\
Quadrupole field gradient, $\partial B / \partial r \partial z\left(\mathrm{~T} / \mathrm{m}^{2}\right)$ & 0.07 \\
Initial solenoid flux density $(\mathrm{T})$ & -3.86 \\
Longitudinal field gradient, $\partial B_{z} / \partial z(\mathrm{~T} / \mathrm{m})$ & 0.54 \\
Peak flux density at conductor $(\mathrm{T})$ & 5.7 \\
Operating current $(\mathrm{kA})$ & 10 \\
Stored energy (MJ) & 4.4 \\
Coil section length along $z$ axis $(\mathrm{mm})$ & 20 \\
Conductor length $(\mathrm{km})$ & 3.3 \\
\hline \hline
\end{tabular}

\section{FACILITY DESCRIPTIONS}

\section{A. Neutrino Factory}

Fig. 4 shows a schematic of a Neutrino Factory. As is obvious, there are many major subsystems that must be combined to create such a facility. The initial segment is a Proton Driver capable of providing a beam power of $4 \mathrm{MW}$. The beam bombards a high-power target that produces pions, which are captured in a solenoidal channel where they decay to muons with a mean momentum of about $200 \mathrm{MeV} / \mathrm{c}$. Following RF manipulations that first turn the single long beam bunch into shorter bunches suitable for a $201 \mathrm{MHz}$ RF system and then increase the bunch duration and decrease the energy spread (longitudinal "phase rotation"), the beam enters an ionization cooling channel, of the type described in Section VI-A. The systems from the target to the cooling channel are referred to collectively as the "Front End."

After leaving the cooling channel, the muon beam is rapidly accelerated to an energy in the range of $20-50 \mathrm{GeV}$ by a linac, followed by two stages of "dogbone" Recirculating Linear Accelerators (RLAs) and finally one or more stages of FixedField Alternating Gradient (FFAG) accelerators. After acceleration to the required energy, the $\mu^{-}$and $\mu^{+}$beams are stored in decay rings having long straight sections oriented toward detectors located roughly $3000 \mathrm{~km}$ and $7500 \mathrm{~km}$ away. 


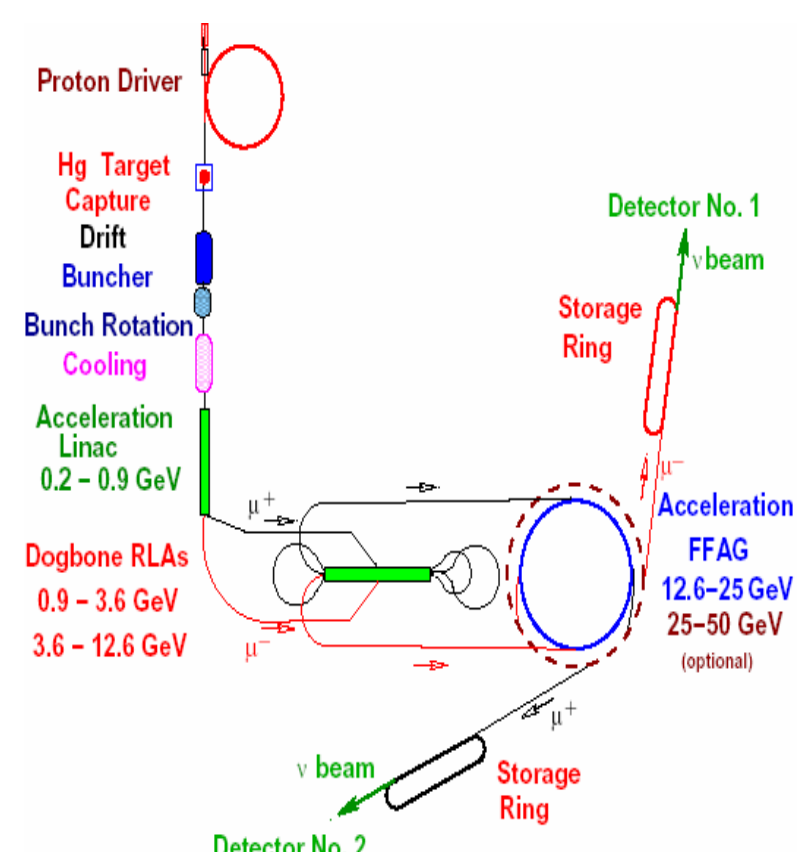

Detector No. 2

Fig. 4. Baseline configuration for a Neutrino Factory developed as part of the International Scoping Study (ISS) [10].

\section{B. Muon Collider}

Fig. 5 shows a schematic layout for a Muon Collider. As can be seen by comparison with Fig. 4, the basic ingredients are mostly the same. There are two main differences between the two types of muon facility. First, the Muon Collider requires $6 \mathrm{D}$ cooling rather than $4 \mathrm{D}$ cooling, because the collider requires a small energy spread to serve as a precision machine. Moreover, the amount of transverse cooling required for the collider is much greater than needed for a Neutrino Factory. Second, the Muon Collider operates at much higher energies than does a Neutrino Factory, roughly $1 \mathrm{TeV}$ rather than a few tens of $\mathrm{GeV}$.

It is worth noting that, because of the basic similarity in the two facilities, much of the ongoing $R \& D$ in support of a Neutrino Factory is equally applicable to a Muon Collider. The main "extra" R\&D required for a Muon Collider focuses on 6D cooling. The proposed MANX experiment [9] is the initial foray into that activity.

Although end-to-end simulations of a Muon Collider have not yet been carried out, progress has been made on simulating the various individual segments. One recent concept for a Muon Collider [11] is based upon utilizing ILC technology for the linac sections of the RLAs. After cooling, the muon beam would be accelerated to about $20 \mathrm{GeV}$ and then the bunches would be coalesced via RF manipulations into fewer, but higher intensity bunches for the collider.

\section{SUBSYSTEM DESCRIPTIONS}

\section{A. Proton Driver}

To deliver roughly $1 \times 10^{21}$ neutrinos per year to the detector, a proton intensity of $4 \mathrm{MW}$ is called for. At a nominal energy of $10 \mathrm{GeV}$, this requires $2.5 \times 10^{15}$ protons per second, i.e., $5 \times 10^{13}$ protons per pulse at a $50 \mathrm{~Hz}$ repetition

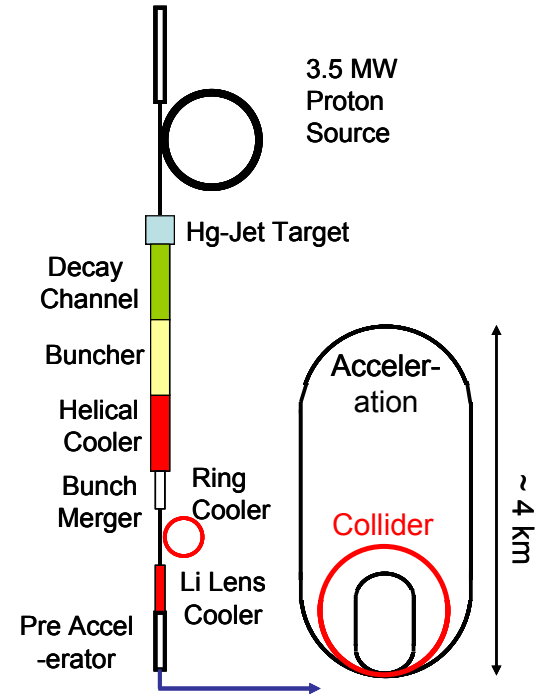

Fig. 5. Schematic of a Muon Collider configuration. Acceleration starts with a linac and is followed by two stages of RLA.

rate. During the course of the ISS, optimization studies of beam energy, bunch length, and accelerator configurations have been carried out. In general, the requirements for the proton driver are not severe, with two exceptions:

1. to retain the ability to do hands-on maintenance on the proton driver, careful control of beam loss is mandatory; and

2. the desire for short bunches $(\approx 2 \mathrm{~ns} \mathrm{rms})$ at a relatively low energy is a challenge.

Various proton driver options have been considered, including a superconducting linac with accumulator and compressor rings, a synchrotron, and an FFAG ring. In practice, any of these options would be acceptable provided it delivers the appropriate proton beam parameters. For this reason, only the parameters of the proton driver have been specified (see Table II), rather than a particular design.

\section{B. Target}

The favored target approach for a Neutrino Factory or Muon Collider is to use a $20 \mathrm{~m} / \mathrm{s}$ liquid-Hg jet, as illustrated in Fig. 6. Although the jet is eventually disrupted by the beam, a fresh portion of the jet is in place when the next beam pulse arrives $20 \mathrm{~ms}$ later. Thus, there is a new target for each beam

TABLE II. PROTON DRIVER SPECIFICATIONS

\begin{tabular}{lc}
\hline \multicolumn{1}{c}{ Parameter } & Value \\
\hline Energy $(\mathrm{GeV})$ & $10 \pm 5$ \\
Beam power $(\mathrm{MW})$ & 4 \\
Repetition rate $(\mathrm{Hz})$ & $\approx 50$ \\
No. of bunch trains & $3,5^{\mathrm{a})}$ \\
Bunch length, rms (ns) & $2 \pm 1$ \\
Beam duration ${ }^{\text {b) }}(\mu \mathrm{s})$ & $\approx 40$ \\
\hline${ }^{\text {a) }}$ Values ranging from 1-5 possibly acceptable. \\
${ }_{\text {b) Maximum spill duration for liquid-metal target. }}$
\end{tabular}


pulse. At the target location there is a $20 \mathrm{~T}$ hybrid solenoid to capture the emitted pions. This field is tapered adiabatically to $1.75 \mathrm{~T}$ over a $20 \mathrm{~m}$ length.

With a $4 \mathrm{MW}$ proton beam, the radiation level in the target area is very high. Nonetheless, it is estimated that the superconducting target magnet will survive in this environment for about 4 years. In this region of the facility, hands-on maintenance is impossible, so all components in Fig. 6 must be designed for remote maintenance and remote replacement.

\section{Cooling Channel}

Several different cooling channels have been studied in recent years [12]-[14]. All contain the same basic components - large bore solenoids, high-gradient RF cavities, and low- $Z$ absorbers. Fig. 7 shows the arrangement used in [13]; this channel is being tested in the MICE experiment [15].

The magnets in Fig. 7 are challenging, but are considered feasible. The main design issue is cost optimization. In a Neutrino Factory, these magnets would likely be cooled by a central cryogenics plant, but for the MICE experiment, individual cryo-coolers will be employed.

The focus coils [15] (see Fig. 8) are being designed by a team from Rutherford Appleton Laboratory and University of Oxford. This magnet has two independently powered coils, which can be run with the same polarity ("solenoid mode") or opposite polarity ("flip mode"). This module will house the $\mathrm{LH}_{2}$ absorbers. Bids for the magnet are presently being evaluated and the contract will be awarded soon.

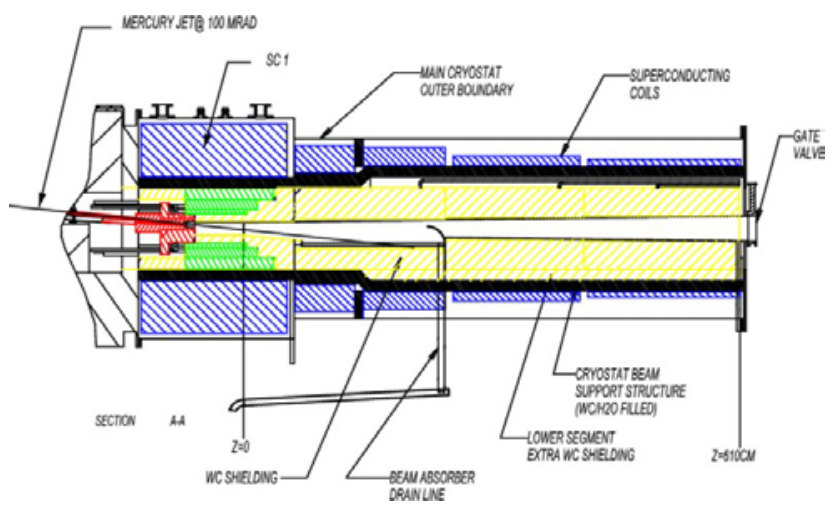

Fig. 6. Diagram of liquid-Hg jet target system. The jet is injected at an angle of $100 \mathrm{mrad}$ with respect to the solenoid axis, and the proton beam enters at an angle of $67 \mathrm{mrad}$. The SC1 magnet is a hybrid, with a superconducting outer coil and a resistive inner coil.

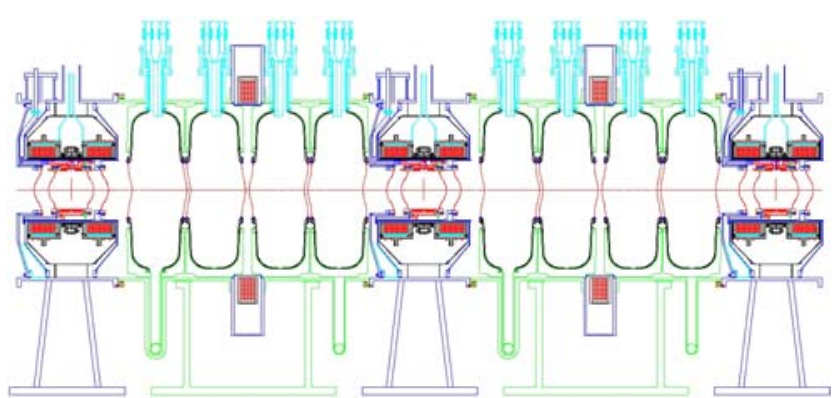

Fig. 7. Diagram of the cooling channel from [13], showing three focus solenoids with $\mathrm{LH}_{2}$ absorbers, eight 201-MHz RF cavities, and two large-bore coupling solenoids placed outside the cavities. This is the channel to be tested in the MICE experiment.
The large diameter coupling coil [16], shown in Fig. 9, was designed by collaborators at LBNL and the Institute of Cryogenics and Superconductivity Technology (ICST) of the Harbin Institute of Technology in China. Because of its large size and the longitudinal constraints resulting from the RF cavity power inputs (see Fig. 7), this magnet is the more challenging of the cooling channel magnets. It has a rather large stored energy, about $9 \mathrm{MJ}$, which means that care must be taken in the design of the quench protection system.

The RF system for a cooling channel represents a substantial challenge. It is based on $201 \mathrm{MHz}$ cavities that are designed to provide a $16 \mathrm{MV} / \mathrm{m}$ accelerating field. R\&D tests on a smaller $805 \mathrm{MHz}$ cavity show a substantial degradation of the achievable gradient when a strong axial magnetic field is applied - exactly the required operating configuration of the cooling channel. A prototype $201 \mathrm{MHz}$ cavity (see Fig. 10) has been built [17] and tested to full gradient without magnetic field at Fermilab. Tests with a realistic magnetic field await the completion of the first coupling coil in about one year.

\section{Acceleration}

Rapid acceleration of the muon beam is one of the most difficult aspects of a Neutrino Factory or Muon Collider. As mentioned earlier, the baseline system from the ISS Neutrino Factory study [10] makes use of a superconducting linac, a pair of dog-bone RLAs, and one or more FFAG rings.

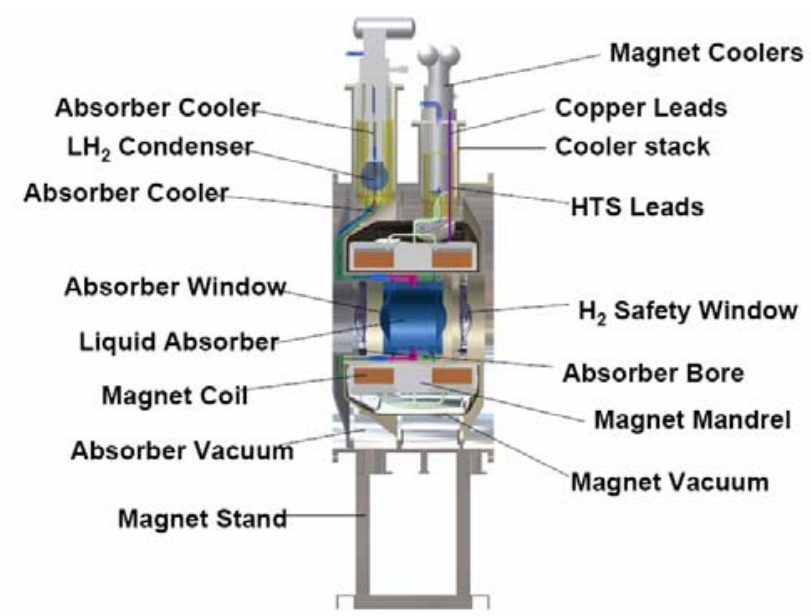

Fig. 8. Side view of focus coil module with an $\mathrm{LH}_{2}$ absorber and its safety windows installed.

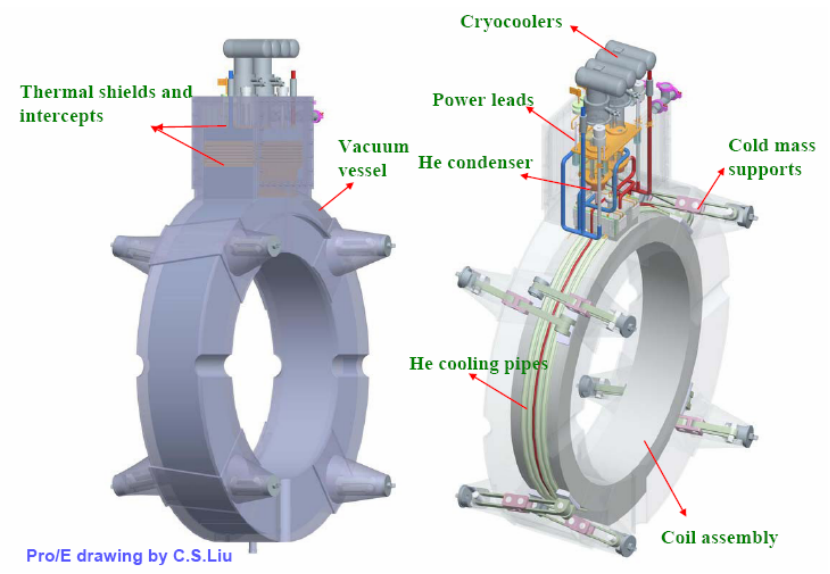

Fig. 9. Coupling coil module design. The coil has an inner diameter of $1.5 \mathrm{~m}$. 


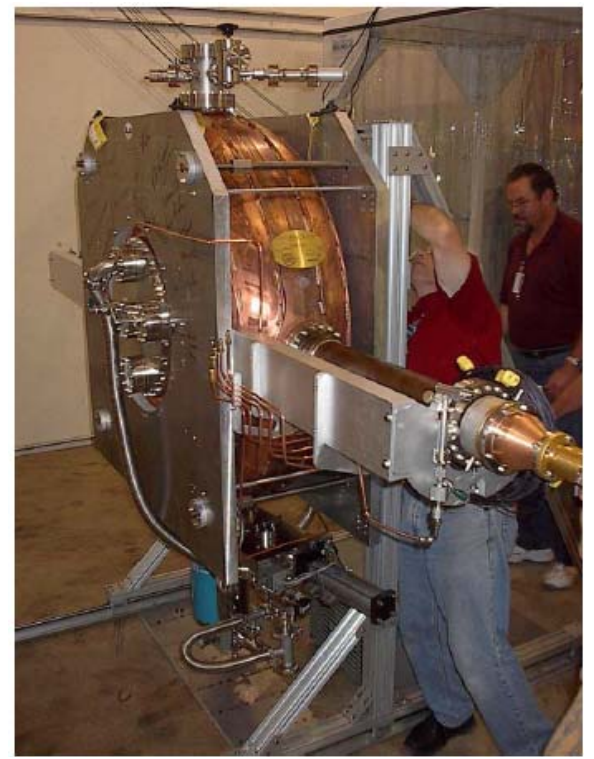

Fig. 10. Prototype $201 \mathrm{MHz}$ RF cavity under test at the MuCool Test Area (MTA) at Fermilab. The cavity easily reached $16 \mathrm{MV} / \mathrm{m}$ gradient in the absence of an external magnetic field.

Because the muon beam is still at low energy in the initial linac, superconducting solenoids are employed for the focusing. The superconducting RF cavities, however, are very sensitive to magnetic fields, so some means of reducing the solenoid fringe fields is needed. A concept for how this might be accomplished is shown in Fig. 11. The idea is to use a superconducting bucking coil outside the main coil, as well as using iron shielding.

The magnetic layout for a typical FFAG ring is illustrated in Fig. 12. The bending magnets are combined-function (dipole plus quadrupole) magnets. A possible design for such a magnet has been developed [18], and is shown in Fig. 13.

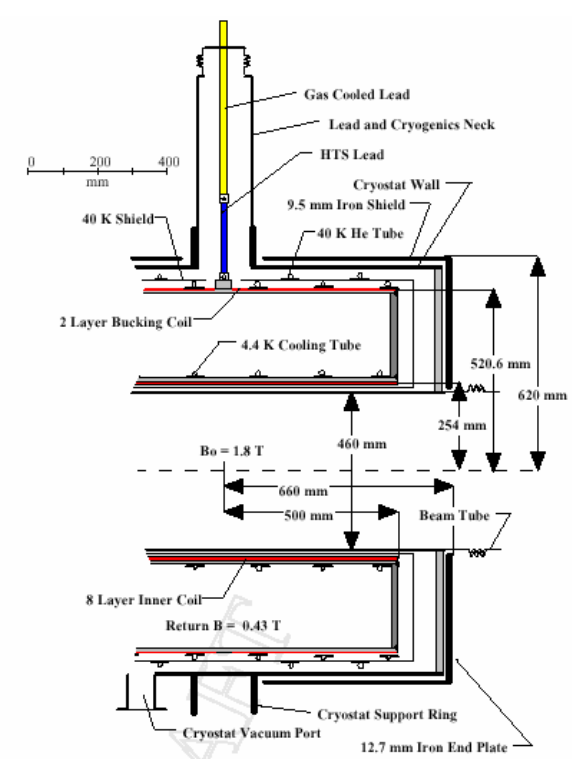

Fig. 11. Side view of shielded superconducting solenoid design suitable for use in the acceleration system initial linac. The nearest RF cavity is located about $1 \mathrm{~m}$ from the end of the magnet.

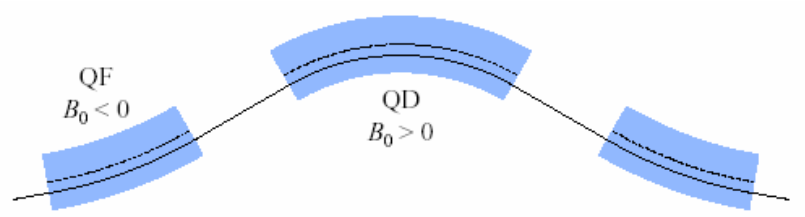

Fig. 12. Layout of one cell of a non-scaling FFAG acceleration ring. The magnet design for this case is illustrated in Fig. 13.

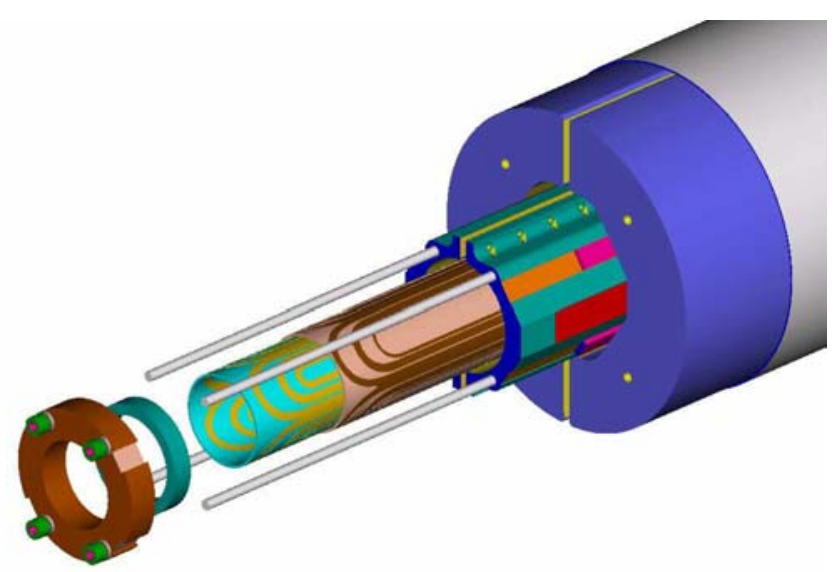

Fig. 13. Design of combined-function magnet suitable for FFAG ring.

\section{E. Decay Ring}

Two different geometries have been studied [19] for the decay rings of a Neutrino Factory-racetrack and triangular. As already noted, the decay ring is specified to be able to send neutrino beams to two detectors at two different baselines, one at about $3000 \mathrm{~km}$ and another at about $7500 \mathrm{~km}$. If the two detector sites are suitably located, the triangular geometry shown in Fig. 14 is optimal. To provide both signs of muon simultaneously, two such rings are needed, as the circulation direction for both rings must be the same.

With the racetrack design, two rings are again required. In this case, each ring is oriented to illuminate one detector. This gives complete flexibility in terms of where the detector sites are located, at the expense of needing two separate tunnels. To make use of both signs of muon, two approaches are possible. The two rings could be injected alternately with $\mu^{-}$and $\mu^{+}$, or each ring could be filled with counter-rotating beams of the two types.

There are two main technical issues to deal with for the decay ring. The first is that the decay electrons from the circulating muon beam create a large heat load in the midplane of the magnets that could result in quenching them. Two approaches are available to handle this. A high- $Z$ inner shield can be used to prevent the decay particles from reaching the magnet coils. This is straightforward, albeit requiring a larger magnet aperture. A second approach that has been considered is to use open-midplane magnets for the decay ring, which allows the power from the decay products to be absorbed at room temperature. 


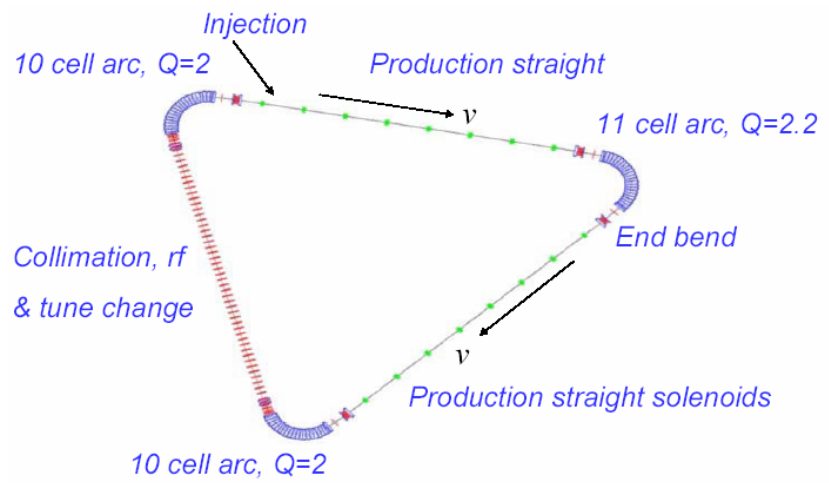

Fig. 14. Layout of a triangular ring that can illuminate detectors at two different baselines. Two such rings, located side-by-side in a common tunnel, would be used to provide time-interleaved beams of $\mu^{-}$and $\mu^{+}$.

\section{R\&D PROGRAM}

$R \& D$ activities are under way in all regions. In Europe, the work is carried out at various institutions and is sponsored by the Beams for European Neutrino Experiments (BENE) organization and the UK Neutrino Factory (UKNF) group. In Japan, work is carried out via the NuFact-J group supported by university funds and some U.S.-Japan high-energy physics funding. Activities in the U.S. are carried out by the Neutrino Factory and Muon Collider Collaboration (NFMCC), sponsored primarily by the Department of Energy with additional support from the National Science Foundation, and joined recently by the Fermilab Muon Collider Task Force (MCTF).

Over time, the work in the various regions has become more international. The MERcury Intense Target (MERIT) test experiment [20], the MICE ionization cooling test [15], the EMMA FFAG program [21], and the International Neutrino Factory Scoping Study [10] are all international efforts.

Here, I will briefly describe some of these ongoing efforts to give an overview of the breadth of the muon beam R\&D activities.

\section{A. MuCool Program}

The MuCool R\&D program [22], part of the NFMCC effort, involves tests of the various components that will comprise an ionization cooling channel, including high gradient normal conducting RF cavities, liquid-hydrogen absorbers, and high-field solenoids. The prototype $201 \mathrm{MHz}$ cavity is shown in Fig. 10. Fig. 15 shows a convection cooled $\mathrm{LH}_{2}$ absorber that was tested with hydrogen in the MTA. Fig. 16 shows the proposed setup for testing the $201 \mathrm{MHz}$ cavity in the magnetic field of a large bore coupling coil when the magnet becomes available next year.

\section{B. Pressurized Cavity Tests}

Tests [23] of a pressurized $805 \mathrm{MHz}$ button cavity (see Fig. 17) are under way in the MTA, led by staff from Muons, Inc. The concept is to use the Paschen effect to limit cavity breakdown. The cavity is filled with high pressure hydrogen gas. Tests to date have been quite successful. In particular, they show gradient limits (Fig. 18) that are unaffected by the presence of a strong magnetic field.

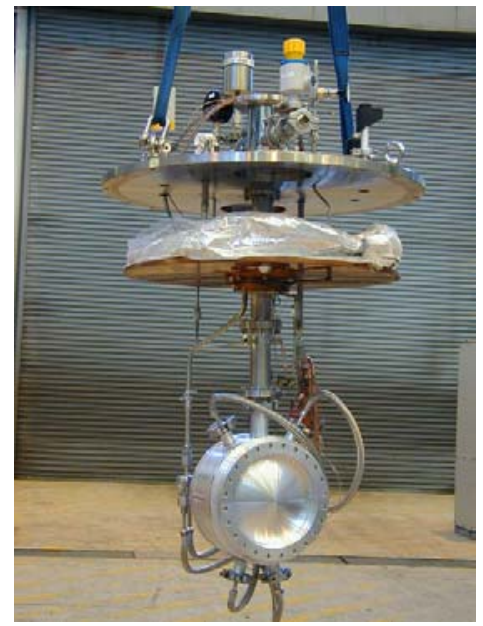

Fig. 15. Body of a liquid-hydrogen absorber being tested at the Fermilab MTA. This device is similar to that to be used in the MICE experiment.

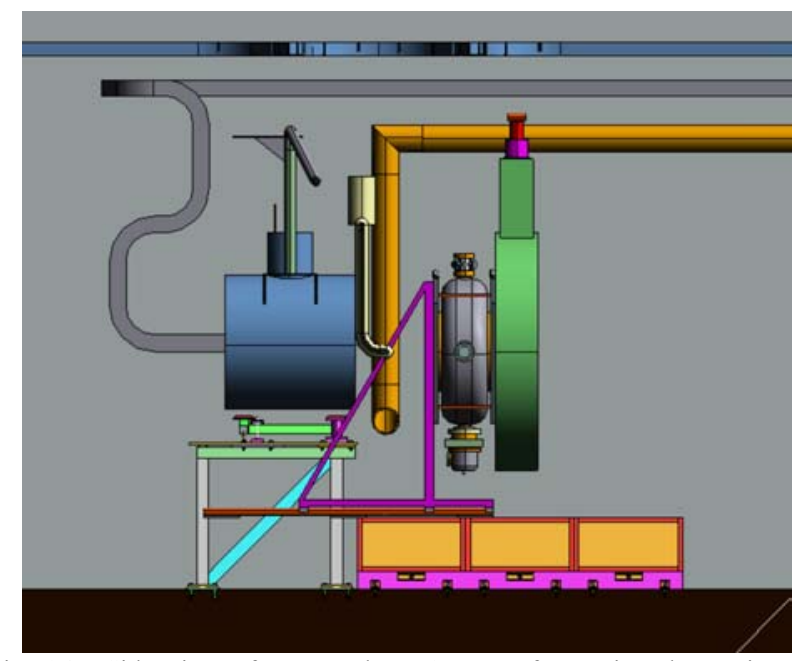

Fig. 16. Side view of proposed MTA setup for testing the cavity with a coupling coil. The rightmost device is the coupling coil, with the $201 \mathrm{MHz}$ cavity immediately to its left. The leftmost device is the existing "Lab G" solenoid that is used to test various $805 \mathrm{MHz}$ cavities within its bore.

There is still one remaining issue to examine for this approach, namely, what happens when an intense beam of ionizing particles traverses the gas-filled cavity. It is possible that this will lead to breakdown in the gas, thus obviating the favorable insulating properties of the gas. Tests of this aspect are planned by the Muons, Inc. group. This will be accomplished by converting the MTA into a beam test facility, using $400 \mathrm{MeV}$ protons from the Fermilab linac.

\section{Ultra-high Field Solenoid Development}

To reach the ultimate emittance required for a Muon Collider, the strongest possible focusing solenoids are needed. The goal of the development program [24] is a device providing $50 \mathrm{~T}$ in a $30 \mathrm{~mm}$ aperture with a length of $1-2 \mathrm{~m}$. It is important to point out that $50 \mathrm{~T}$ is a design goal, but not a firm requirement. That is, there is no hard cutoff on the strength of these magnets - the aim is simply to produce the highest practical strength. The plan is to first focus on materials, particularly high-temperature superconductors (HTS), with the aim of eventually fabricating a 25-30 T test model. 


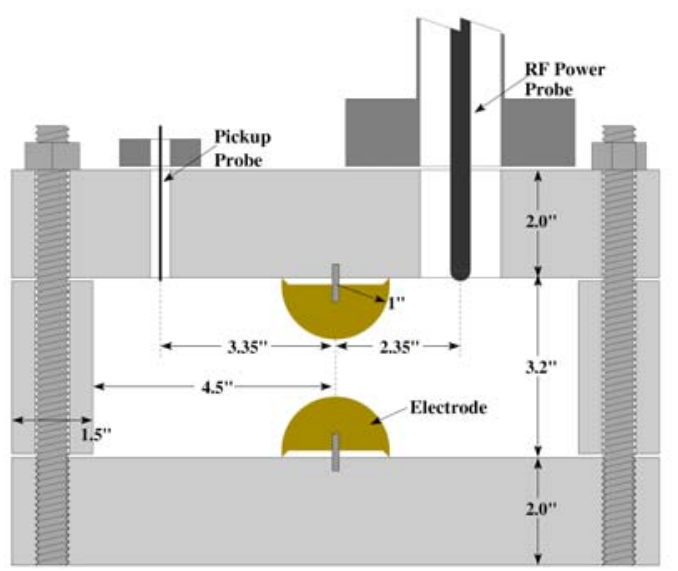

Fig. 17. Diagram of pressurized $805 \mathrm{MHz}$ pillbox cavity to test the influence of hydrogen gas in preventing breakdown. The button electrodes can be fabricated from various materials to test their properties. Thus far, tests with $\mathrm{Cu}, \mathrm{Be}$, and Mo electrodes have been carried out.

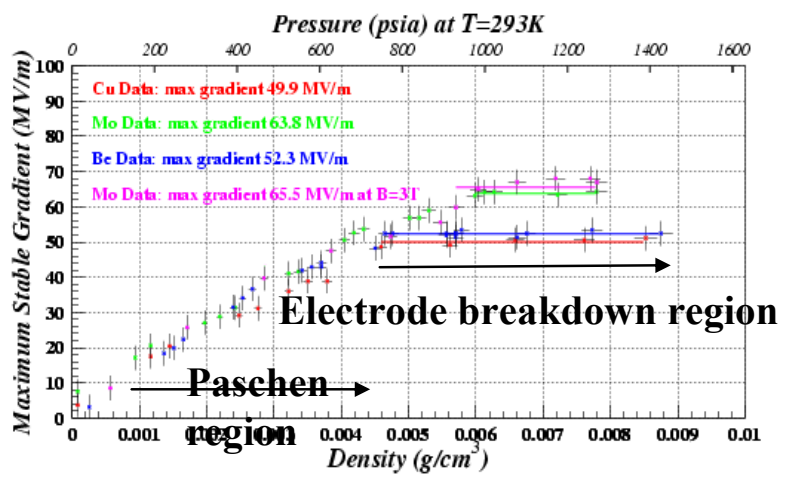

Fig. 18. Results from the pressurized cavity shown in Fig. 17. The allowable gradient increases linearly with pressure (measured in terms of gas density) until reaching a limit dictated by the electrode material. The two upper lines both represent Mo electrodes, one with and one without a superimposed magnetic field; there is no degradation in maximum gradient in these tests. The two lower lines are for $\mathrm{Cu}$ and $\mathrm{Be}$ electrodes, with Be achieving the slightly higher gradient.

\section{Target Tests}

The test of a Hg-jet target in a $15 \mathrm{~T}$ solenoid is the goal of the MERIT experiment [20], an international experiment being mounted at CERN. Fig. 19 shows the concept and Fig. 20 shows the actual apparatus installed at CERN. Looking at Fig. 19, at the left there is a high-pressure syringe pump to create the $\mathrm{Hg}$ jet. The $15 \mathrm{~T}$ magnet is to the right. This magnet is cryogenic but not superconducting. It comprises three nested coils and operates at liquid-nitrogen temperature to reduce the requirements on the power supply. After each pulse, the magnet must be recooled, so it will pulse at most only $2-3$ times per hour. First beam for the experiment is scheduled for October 2007.

\section{E. $M I C E$}

The international MICE experiment [15] will provide the first experimental verification of muon ionization cooling. The muons will be generated parasitically from the circulating $800 \mathrm{MeV}$ proton beam from the ISIS synchrotron at RAL. First beam is expected in February 2008. As described earlier, the experiment will test one cell of a realistic cooling channel,

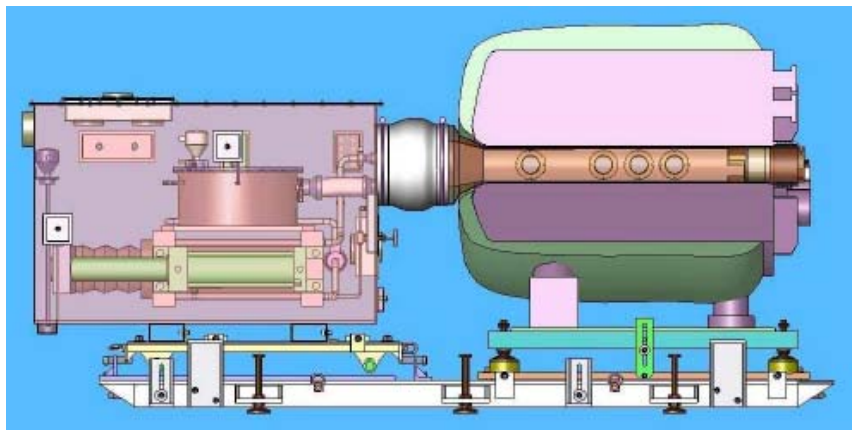

Fig. 19. Drawing of MERIT experiment. The syringe pump and $\mathrm{Hg}$ containment are shown on the left, with a tube connecting the $\mathrm{Hg}$ jet to the bore of the $15 \mathrm{~T}$ solenoid. Optical diagnostics are used at the viewports to determine what happens to the jet when hit by a $24 \mathrm{GeV}$ beam from the CERN PS. The beam and the jet will both travel from right to left in this picture.

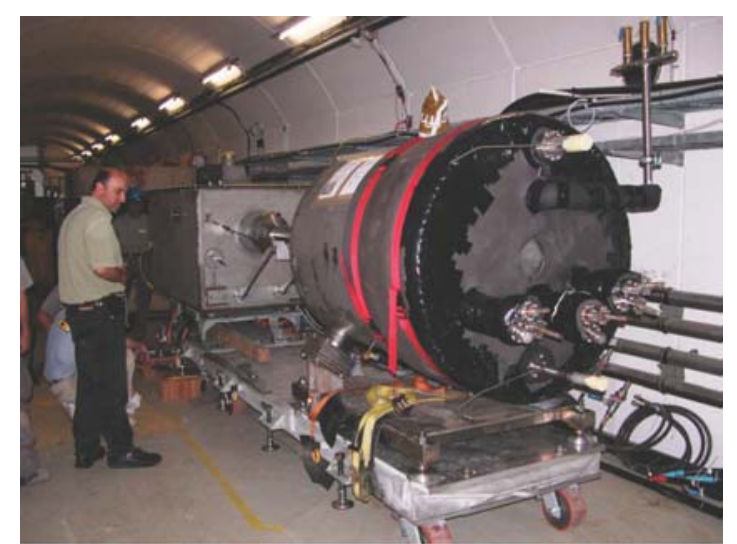

Fig. 20. Photograph of the MERIT apparatus installed in the TT2A tunnel at CERN. The $15 \mathrm{~T}$ solenoid is in the foreground with the $\mathrm{Hg}$ containment located downstream.

using spectrometers upstream and downstream to measure the emittance particle by particle. The aim is to measure a $10 \%$ cooling effect with a precision of $0.1 \%$.

As indicated in Section VIII-C, most components for MICE are in the final design stages, and many are already in production. The experiment will be carried out in stages to minimize the influence of systematic errors on the results. The first two stages, beginning next year, will characterize the muon beam and compare the two spectrometer systems without the intervening cooling channel. Thereafter, a single absorber and focus coil module will be added, followed by a second absorber and focus coil module and the first RFCC module. The last stage, planned to be completed in 2010, will test the full unit cell shown in Fig. 7.

\section{F. MANX}

The MANX experiment [9], still in the planning stage, is envisioned to run at Fermilab. This experiment will test the concept of 6D cooling with continuous emittance exchange (see Section VI-B) in a helical cooling channel. A simulation of the channel is shown in Fig. 21. The predicted cooling is roughly $30 \%$ in each phase plane, leading to an overall reduction in $6 \mathrm{D}$ emittance by about a factor of two. For this initial experiment, no RF cavities will be utilized. However, cavities will eventually be needed in an actual cooling channel and will need to be incorporated in the test channel eventually. 


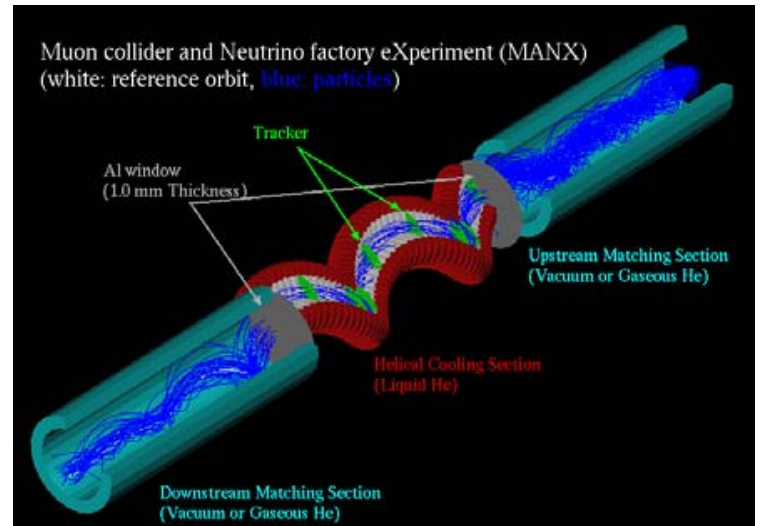

Fig. 21. Simulation of a 6D cooling experiment, MANX. The beam is matched into and out of the $3.2 \mathrm{~m}$ helical cooling channel with solenoidal matching sections. The central portion of the channel will employ liquid helium rather than liquid hydrogen to mitigate safety concerns.

\section{Summary}

R\&D toward the design of intense muon beam facilities is progressing on many fronts, though there is still much to be done. Key areas include the high-power target and the ionization cooling channel. The need for rapid acceleration has led to the rediscovery of the FFAG concept and has given rise to a number of interesting technical issues. A substantial R\&D program is needed because the design challenges of a muon accelerator complex go well beyond those of standard beams. This program must address the issues of expected performance, technical feasibility and technical risk, and also cost. To ensure a realistic approach, it is paramount that the R\&D program include experiments and the building of actual components to test. Paper studies alone are not enough.

Intense muon facilities have great potential to address some of the critical outstanding questions in particle physics, including the origins of the matter-dominated universe and the origins of mass. Their design, though challenging, is clearly worthy of the attention of the scientific community.

As with all new accelerator developments, success depends on a synergy between accelerator physics and accelerator technology. In particular, interesting and challenging magnets play a role in both Neutrino Factory and Muon Collider designs. As has been true in the past, the skills of the magnet designers and builders will be critical to turning the dreams of the accelerator physics community into the cutting-edge scientific tools of the future.

\section{ACKNOWLEDGMENT}

I would like to thank my accelerator physics and particle physics colleagues in the NFMCC and around the world who participate so energetically in this challenging endeavor. It should be obvious that the substantial R\&D progress reported here is due to their efforts. I would also like to thank the many engineers who are contributing to this exciting activity, without whom little, if anything, would ever get built. In particular, Mike Green, Steve Virostek, and Wing Lau have taught me a lot about what is possible and affordable, andmore importantly-what is not. Finally, I would like to thank DOE and NSF for having the vision-even in difficult financial times - to support the advanced accelerator R\&D activities that provide future choices for particle physics.

\section{REFERENCES}

[1] J. H. Christenson, J.W. Cronin, V. L. Fitch, and R. Turlay, "Evidence for the $2 \pi$ decay of the $K_{2}^{0}$ meson," Phys. Rev. Lett., vol 13, pp. 138-140, Jul. 1964.

[2] M. S. Zisman (ed.), "PEP-II, an asymmetric B factory, conceptual design report," LBL-PUB-5379, SLAC-418, CALT-68-1869, UCRL-ID114055, UC-IIRPA-93-01, June, 1993.

[3] S. Kurokawa and K. Oide (eds), "KEKB B-factory design report," KEK report 95-7, June 1995.

[4] "LHC design report," [Online], http://ab-div.web.cern.ch/abdiv/Publications/LHC-DesignReport.html.

[5] D. Degèle, "The HERA e-p collider," in Proc. 1992 European Particle Accelerator Conference, Berlin, Germany, March 24-28 1992, pp. 3-8.

[6] N. Phinney, N. Toge, and N. Walker (eds.), "ILC reference design report," [Online], http://ilcdoc.linearcollider.org/getfile.py?docid= 182\&name=ILC_RDR_Volume_3-Accelerator\&format=pdf.

[7] S. F. King, K. Long, Y. Nagashima, B. L. Roberts, and O. Yasuda (eds.), "Physics at a future neutrino factory and super-beam facility," [Online], http://www.hep.ph.ic.ac.uk/\%7Elongkr/UKNF/Scoping-study/ISSwww-site/WG1-PhysPhen/Planningdrafts/Report/Current/PhysReport.pdf, July, 2007.

[8] V. S. Kashikhin, et al., "Superconducting Helical Solenoid for Muon Cooling Experiment at Fermilab," paper 4V04, these proceedings.

[9] K. Yonehara, et al., "The MANX muon cooling demonstration experiment," in Proc. 2007 Particle Accelerator Conf., Albuquerque, June 25-29, 2007, pp. 2969-2971.

[10] M. S. Zisman (ed.), "Summary Report of Accelerator Working Group," [Online]. Available: http://www.cap.bnl.gov/mumu/project/ISS/ISSAccelerator WGbrief-R2a.pdf.

[11] K. Paul, R. P. Johnson, and V. Yarba, "Summary of low-emittance muon collider workshop (February 6-10, 2006)," in Proc. of 2006 European Particle Accelerator Conf., Edinburgh, Scotland, June 26-30, 2006, pp. 2412-2414.

[12] N. Holtkamp and D. Finley (eds.), "A feasibility study of a neutrino source based on a muon storage ring," Fermilab Pub-00/108E, [Online]. Available : http://www.fnal.gov/projects/muon_collider/nu-factory/ fermi_study_after_aprillst/.

[13] S. Ozaki, R. Palmer, M. Zisman, and J. Gallardo (eds.), "Feasibility study-II of a muon-based neutrino source," BNL-52623, June, 2001, [Online]. Available: http://www.cap.bnl.gov/mumu/studyii/final_ draft/The-Report.pdf.

[14] J. S. Berg et al., "Cost-effective design for a neutrino factory," Phys. Rev. Special Topics-AB, vol. 9, 011001 (2006).

[15] P. Drumm (ed.), "MICE: an international muon ionization cooling experiment, Technical design report, [Online]. Available: http://www.isis.rl.ac.uk/accelerator/MICE/TR/MICE_Tech_ref.html.

[16] M. A. Green et al., "Progress on the design of the coupling coils for MICE and MuCool," in Proc. 2007 Particle Accelerator Conf., Albuquerque, June 25-29, 2007, pp. 500-502.

[17] D. Li et al., "201 MHz cavity R\&D for MuCool and MICE," in Proc. of 2006 European Particle Accelerator Conf., Edinburgh, Scotland, June 26-30, 2006, pp. 1367-1369.

[18] S. Caspi and R. Hafalia, LBNL report SC-MAG-839, 2004 (unpublished).

[19] G. Rees, C. Johnstone, and F. Méot, "20-50 GeV muon storage rings for a neutrino factory," in Proc. of 2006 European Particle Accelerator Conf., Edinburgh, Scotland, June 26-30, 2006, pp. 2415-2417.

[20] H. G. Kirk et al., "A high-power target experiment at the CERN PS," in Proc. 2007 Particle Accelerator Conf., Albuquerque, June 25-29, 2007, pp. 646-648.

[21] R. Edgecock, "EMMA - the world's first non-scaling FFAG," in Proc. 2007 Particle Accelerator Conf., Albuquerque, June 25-29, 2007, pp. 2624-2626.

[22] J. Norem et al., "Recent RF results from the MuCool test area," in Proc. 2007 Particle Accelerator Conf., Albuquerque, June 25-29, 2007, pp. 2239-2241.

[23] P. Hanlet et al., "High pressure RF cavities in magnetic fields," in Proc. of 2006 European Particle Accelerator Conf., Edinburgh, Scotland, June 26-30, 2006, pp. 1364-1366.

[24] V. V. Kashikhin, E. Barzi, V. S. Kashikhin, M. Lamm, and A. V. Zlobin, "Study of high field superconducting solenoids for muon beam cooling," paper 4L01, these proceedings. 